\title{
Thanks to Reviewers
}

The Editor-in-Chief and Associate Editors of the European Journal of Medical Physics wish to express their appreciation to the following persons who acted as reviewers over the last 12 months.

A

Hilal Acar (Turkey)

Jean-François Adam (France)

Pedro Almeida (Portugal)

Peter Anderson (United States)

Marta Anguiano (Spain)

Hidetaka Arimura (Japan)

Rafael Arrans (Spain)

Yoshiyuki Asai (Japan)

\section{B}

Dale L. Bailey (Australia) Colin Baker (United Kingdom)

Facundo Ballester (Spain)

Fabrizio Banci Buonamici (Italy)

Maurizio Baroni (Italy)

Grzegorz Bartosz (Poland)

Enrique Berjano (Spain)

Lorenzo Bianchi (Italy)

Peter John Biggs (United States)

Pierre-yves Bondiau (France)

Faiza Bourhaleb (Italy)

Marco Brambilla (Italy)

Gunnar Brix (Germany)

Marta Bucciolini (Italy)

\section{C}

Jose Perez Calatayud (Spain) Alfonso Calzado Cantera (Spain)

C.J. Caruana (Malta)

Carlo Cavedon (Italy)

Gunther Christ (Germany)

Marianerina Cinti (Italy)

Mario Ciocca (Italy)

Maurizio Conti (United States)

Ana V. Coronado (Spain)

Remo Crescenti (Switzerland)

Giacomo Cuttone (Italy)
D

Cinzia da via (United Kingdom)

Tommaso D'Alessio (Italy)

Yves D’Asseler (Belgium)

Mario de Denaro (Italy)

Victor Delgado (Spain)

Pasquale Delogu (Italy)

Güleser Kalay Demir (Turkey)

Slobodan Devic (Canada)

Rohan Dharmakumar (United States)

Oliver Dohm (Germany)

Christian Drexler (Germany)

Hanlie Du Raan (South Africa)

Peter Dunscombe (Canada)

E

Wolfgang Enghardt (Germany)

Tülay Ercan (Turkey)

$F$

Dario Faj (Croatia)

Allan G. Farman (United States)

Keith Faulkner (United Kingdom)

Claudio Fiorino (Italy)

Alfredo Fiume (Italy)

Malte Frese (Germany)

Gregory Fridman (United States)

Christer Frojdh (Sweden)

G

Marco Galelli (Italy)

Gisella Maria Gennaro (Italy)

Philippe Giraud (France)

Damian Guirado (Spain)

H

Bin Han (United States)

Christine Harting (Germany)
Bijan Hashemi (Islamic Republic of Iran) Walter Huda (United States)

$J$

Vesna Jacob (Germany)

Walter Jentzen (Germany)

José Ramón Jiménez (Spain)

Martti Juhola (Finland)

Jaroslav Julak (Czech Republic)

K

S. Kameoka (Japan)

Christian P. Karger (Germany)

Christof Karmonik (United States)

Ulrich Kertzscher (Germany)

Sakae Kinase (Japan)

Christian Kirisits (Austria)

Herbert Köstler (Germany)

Tomas Kron (Australia)

Stephen Kry (United States)

Yang Kuang (United States)

$L$

Fedele Laitano (Italy)

Paul Lecoq (Switzerland)

John Lee (Belgium)

Pei-Jan Paul Lin (United States)

Albert Lisbona (France)

Maria do Carmo Lopes (Portugal)

stefano luin (Italy)

M

Jürgen Machann (Germany)

Julian Malicki (Poland)

Eirik Malinen (Norway)

sara marcatili (Italy)

Colin J. Martin (United Kingdom)

Maria Martisikova (Germany)

Arnulf Materny (Germany) 
Ruth McLauchlan (United Kingdom) $\quad R$

Ali S. Meigooni (United States)

Ana Maria Mendonca (Portugal)

Moyed Miften (United States)

Peter Mohr (Germany)

domenico mola (Italy)

Anargyros Moutsatsos (Greece)

\section{$N$}

Suzanne Naudy (France)

Heidi Nettelbeck (Australia)

Bo Nilsson (Sweden)

Raffaele Novario (Italy)

Hakan Nystrom (Sweden)

0

Tomohisa Okuma (Japan)

Daniela Origgi (Italy)

\section{$P$}

Renato Padovani (Italy)

Marco Pagani (Italy)

Hugo Palmans (United Kingdom)

George Panayiotakis (Greece)

Antonios E. Papadakis (Greece)

Manuel A. Parafita (Spain)

Juan Pardo Montero (Italy)

Renzo Perfetti (Italy)

Daniel Pflugfelder (Germany)

luisa pierotti (Italy)

Jaroslaw Piskorski (Poland)

Emily Poon (Canada)

Björn Poppe (Germany)

Richard Popple (United States)

Ambika Pradhan (Republic of Korea)

Q

Sima Qamhiyeh (Germany)
$R$

Magdalena Rafecas (Spain)

Osvaldo Rampado (Italy)

Alfonsina Ramundo Orlando (Italy)

Sabine Reinhardt (Germany)

Eric Reynard (Canada)

Christian Richter (Germany)

Cesare Romagnoli (Canada)

Jean-Claude Rosenwald (France)

Chun Ruan (United States)

Mark Ruschin (Canada)

Paolo Russo (Italy)

Eva Rutkowska (United Kingdom)

\section{$S$}

Dariush Sardari (Islamic Republic of Iran)

Otto Sauer (Germany)

Andrea Schaefer-Schuler (Germany)

Marco Schippers (Switzerland)

Anthony Seibert (United States)

Chengyu Shi (United States)

Frank-Andre Siebert (Germany)

Jan Sijbers (Belgium)

José Silvestre Silva (Portugal)

Annie Simon (United States)

Parjit Singh (India)

Katarina Sjogreen-Gleisner (Sweden)

Steven Sourbron (United Kingdom)

Angelique Stephanou (France)

Edmond Sterpin (Belgium)

Markus Stock (Austria)

Ferdinand Sudbrock (Germany)

Justin Sutherland (Canada)

$T$

Slavik Tabakov (United Kingdom)

Karsten Tabelow (Germany)

Angelo Taibi (Italy)

Akihiro Takaki (Japan)

Michael Taylor (Australia)

Lena Tirpak (United States)
Benjamin Titz (United States)

Milan Tomsej (Belgium)

Paula Toroi (Finland)

Javier Torres (Spain)

Kalman Toth (Hungary)

loannis A. Tsalafoutas (Greece)

V

Wil van der Putten (Ireland)

Wouter van Elmpt (Netherlands)

Wim van Hecke (Belgium)

Gerard C. van Rhoon (Netherlands)

Marcel van Straten (Netherlands)

Stijn Vandenberghe (Switzerland)

Eliseo Vano (Spain)

Peter Vaupel (Germany)

Francis R. Verdun (Switzerland)

Rosa Villa (Spain)

Stefaan Vynckier (Belgium)

W

Jing Wang (United States)

Colin A. Webber (Canada)

René Werner (Germany)

Hans Wiksell (Sweden)

Florian Wolf (Austria)

Michael Wucherer (Germany)

$X$

X. George Xu (United States)

Y

Fu-Hua Yan (China)

Kai Yang (United States)

Füsun Yasar (Turkey)

Z

Luis I. Zamora (Spain)

Bin Zheng (United States)

Piotr Zygmanski (United States) 